\title{
Painful right elbow mass
}

\author{
Veronica Attard $^{1}$ (D) - Asif Saifuddin ${ }^{1}$
}

Published online: 13 December 2018

(C) ISS 2018

Diagnosis: Neurolymphoma of the median nerve.

\section{Discussion}

Neurolymphoma (NL) is a rare entity representing peripheral nervous system infiltration by malignant lymphocytes [1]. It is most commonly seen in the context of aggressive sub-types of lymphoma, such as diffuse large B cell non-Hodgkin's lymphoma [2]. Its exact incidence is not well documented [3].

While only $20 \%$ of patients with NL have evidence of systemic involvement at the time of staging, systemic disease is commonly reported at autopsy [4]. Involvement of peripheral nerves without systemic manifestations, i.e. primary neurolymphomatosis, has been most commonly reported within the sciatic nerve [5]. Clinical presentation is variable and includes cranial neuropathy, painful and painless mononeuropathy, painful radiculopathy, and polyneuropathy [3].

The diagnosis of NL hinges upon both radiological and histopathological examination, in view of its rarity and diverse clinical presentation. NL most frequently involves the proximal peripheral nerves and brachial and lumbosacral plexuses. Imaging appearances range from diffuse enlargement of the peripheral nerve to a mass-like appearance of the involved nerve [2]. NL demonstrates T2 hyperintensity and a nodular pattern or fusiform enlargement [4]. T2-weighted imaging usually demonstrates scattered areas of hypointensity within the mass in keeping with displaced nerve fascicles within the tumor. MRI features that suggest a diagnosis of NL include

The case presentation can be found at https://doi.org/10.1007/s00256$018-3125-\mathrm{z}$

Veronica Attard

veronica2687@gmail.com

1 Radiology Department, Royal National Orthopaedic Hospital, Stanmore, UK enlargement of nerves or nerve roots beyond the root sleeve [1]. Post-contrast imaging may show homogenous or heterogeneous enhancement of the mass lesion [5]. Ultrasonographic features of NL are very different from peripheral neurogenic tumors. In NL, sonographic features include infiltration of the nerve by a surrounding mass, focal thickening of the nerve or of individual fascicles within the nerve. Nerve biopsies may yield false-negative results due to the patchy distribution of NL [4].

The differential considerations include peripheral spread of malignant disease. Perineural spread of malignancy may occur in head and neck tumors, gastrointestinal malignancy, breast cancer, prostate cancer, and cervical cancer. Other differential considerations of NL include inflammatory neuropathy. At MR imaging, inflammatory neuritis is depicted as diffuse thickening of the affected nerves and returns a high T2 signal. Peripheral nerve sheath tumors can mimic NL, however, the former appear heterogenous at MR imaging. Peripheral nerve sheath tumors return a low signal intensity on T1-weighted imaging and intermediate to high signal on T2-weighted imaging [4]. Differential diagnoses in patients with a prior history of lymphoma includes lymphoma-associated vasculitis and amyloidosis [4].

In our case, MRI showed a fusiform soft tissue mass extending from the inferior portion of the right upper arm and crossing the elbow joint (Fig. 1a, b). The mass measured $16 \mathrm{~cm}$ in craniocaudal dimension with a maximum crosssectional dimension of $4.6 \times 3 \mathrm{~cm}$. The mass appeared to arise from the median nerve, which itself was noted to be thickened. The mass interdigitated with the adjacent nerve fibers (Fig. 1b, c) and displaced the adjacent brachial artery. It demonstrated intermediate to low signal intensity (SI) to adjacent muscle on T1 (Fig. 1a) and high SI on T2 (Fig. 1b) and fat-suppressed imaging (Fig. 1c). Multiple dot-like dark signal foci were noted on proton density and fat-suppressed T2-weighted imaging (Fig. 1c) consistent with displaced nerve fascicles in the tumor. These foci were depicted as low signal striations along the course of the nerve on coronal and sagittal imaging (Fig. 1b). The T2 hyperintensity and enlargement of the nerves were possible indicators of malignancy [4]. 
An additional lesion of similar SI was also present more inferiorly within the muscle belly of the common flexor tendons. The lesion measured $6.8 \mathrm{~cm}$ with a maximum crosssectional diameter of $2 \times 2 \mathrm{~cm}$. Gadolinium was not administered in this case due to mild renal impairment.

US showed a heterogeneous mass sited in the anteromedial aspect of the right antecubital fossa (Fig. 2). It had a slightly hyperechoic central core, representing its main neural component.

Microscopic examination revealed a tumor with a sheathlike architecture composed of lymphoid cells with a high nuclear:cytoplasmic ratio and hyperchromasia. Atypical mitoses were also noted. Immunohistochemistry confirmed that the tumor cells were positive for CD20. The patient was referred to hematooncology for further management.

Fluorine $18\left({ }^{18} \mathrm{~F}\right)$ fluorodeoxyglucose (FDG) positron emission tomography (PET)/computed tomography (CT) demonstrated areas of increased metabolic activity along the enlarged median nerve. No other areas of uptake were identified.

The prognosis of patients with NL is bleak despite treatment, probably relating to current lack of standardized regimens [3]. Our patient was treated with standard chemotherapy with CNS prophylaxis, and the mass demonstrated positive therapeutic response within a few months.

In summary, we report a rare case of primary NL involving the median nerve. MRI usually demonstrates diffuse enlargement of the peripheral nerve or mass formation along the course of the involved nerve. Characteristic MRI features such as tumor homogeneity despite large tumor size, and preservation of adjacent structures including interdigitating nerve fascicles, may be useful imaging features in differentiating NL from other soft tissue tumors [3]. NL can be misdiagnosed as a neurogenic tumor on imaging. Our case demonstrated linear structures that continued to the nerve fascicles along the fusiform lesion on coronal and sagittal images. The contour of the involved nerve was preserved, which helps to distinguish NL from other malignant peripheral nerve sheath tumors. The latter tend to be heterogeneous in appearance and result in destruction of the nerve architecture [5]. A combination of various imaging modalities and image-guided biopsy are of utmost importance in obtaining the final diagnosis and facilitating further management.

Authors' contributions VA wrote the manuscript. All authors reviewed the final manuscript.

\section{Compliance with ethical standards}

Conflict of interest The authors declare that they have no conflicts of interest.

\section{References}

1. Shree R, Goyal M, Modi M, Gaspar B, Radotra B, Ahuja C, et al. The diagnostic dilemma of neurolymphomatosis. J Clin Neurol. 2016;12(3):274.

2. Caliskan B, Bonyadlou S, Peterson J. Positron emission tomography/ computerized tomography imaging of multiple focus of neurolymphomatosis. IJNM. 2014;29(4):252.

3. Lee J, An J, Park J, Ryu K, Moon S. Primary Neurolymphoma of the Tibial nerve: a case report with characteristic MRI findings. JKSR. 2016;75(5):399.

4. Crush A, Howe B, Spinner R, Amrami K, Hunt C, Johnson G, et al. Malignant involvement of the peripheral nervous system in patients with cancer: multimodality imaging and pathologic correlation. Radiographics. 2014;34(7):1987-2007.

5. Wadhwa V, Thakkar R, Maragakis N, Höke A, Sumner C, Lloyd T, et al. Sciatic nerve tumor and tumor-like lesions - uncommon pathologies. Skelet Radiol. 2012;41(7):763-74. 